\title{
Amyotrophic Lateral Sclerosis
}

\author{
Toshiyuki Araki, MD, PhD \\ Editor
}

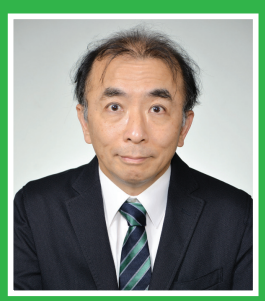

Toshiyuki Araki, MD, PhD, is Director of the Department of Peripheral Nervous System Research at the National Institute of Neuroscience of the National Center of Neurology and Psychiatry (Tokyo, Japan), visiting professor at Tokyo University of Agriculture and Technology (Tokyo, Japan), and lecturer at Waseda University (Tokyo, Japan) and Osaka University (Osaka Japan). He graduated from Osaka University, where he received MD and PhD degrees. He performed postgraduate training at the Washington University School of Medicine in St. Louis (Missouri, USA). The research interest of Dr. Araki has been focused on the neuronal/axonal degeneration mechanisms and application of the knowledge to the therapeutic development. The targets of his research range from peripheral nerve degeneration/regeneration and neuromuscular disorders to neurodegenerative disorders including amyotrophic lateral sclerosis and Alzheimer's disease, and developmental disorders including autism-spectrum disorders. For promoting translational research, he has been collaborating with several pharmaceutical companies. He also serves as the chairman of National Center of Neurology and Psychiatry Ethics Committee for clinical research, and an ethical advisor to Brain/Minds Beyond project. He has authored more than 100 scientific publications. He also serves as an editorial member of scientific journals, and a board member/ councilor of several scientific societies.
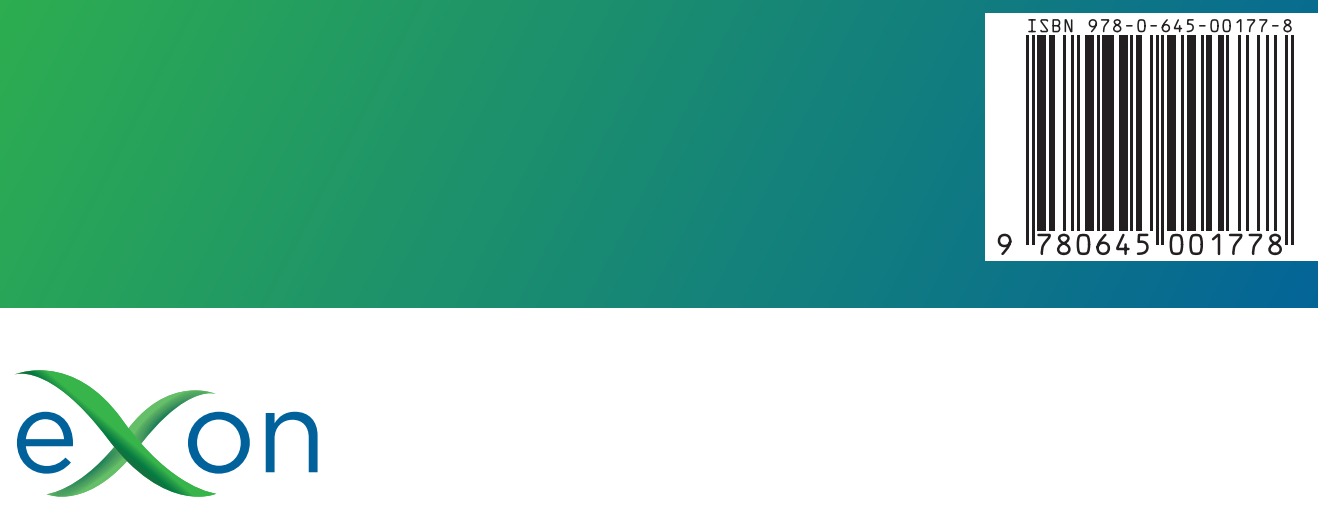

PUBLICATIONS 